CVIA

CASE REPORT

pISSN 2508-707X / elSSN 2508-7088 https://doi.org/10.22468/cvia.2017.00080 CVIA 2017;1(4):248-250

Received: May 15, 2017

Revised: August 10, 2017

Accepted: September 21, 2017

Corresponding author

Rositaa Mohd Ibrahim, MD, MRad

Department of Diagnostic Imaging,

Hospital Serdang, Jalan Puchong,

43000 Kajang, Selangor, Malaysia

Tel: 60-1-2429-0495

Fax: 60-3-8947-5322

E-mail: drositaa@yahoo.com

\section{Typical and Atypical Magnetic Resonance Imaging Appearances of Primary Cardiac Lymphoma}

\author{
Rositaa Mohd Ibrahim', Mohamad Syafeeq Faeez Md Noh², \\ Mohamad Arif Muhammad Nor ${ }^{3}$, Nor Ashikin Md Sari ${ }^{4}$, \\ Norafida Bahari², Ezamin Abd Rahim², Yusri Mohammed ${ }^{1}$ \\ 'Department of Diagnostic Imaging, Hospital Serdang, Malaysia \\ ${ }^{2}$ Department of Imaging, Faculty of Medicine and Health Sciences, Universiti Putra, Malaysia \\ ${ }^{3}$ Department of Cardiothoracic Surgery, Hospital Serdang, Malaysia \\ ${ }^{4}$ University Malaya Medical Centre, Kuala Lumpur, Malaysia
}

\begin{abstract}
Primary cardiac lymphoma (PCL) accounts for $1.3 \%$ of all cardiac tumours. Diagnosis is particularly challenging owing to its rarity and non-specific presentation. Although transthoracic echocardiogram is the initial imaging modality used to detect cardiac masses, advances in cardiac magnetic resonance imaging (CMR) has allowed more detailed assessment, which enables accurate diagnosis and subsequent therapy. We would like to highlight two cases of PCL that were diagnosed in our institution, manifesting with typical and atypical CMR features.
\end{abstract}

Key words Cardiac lymphoma · Cardiac imaging · Cardiac tumour.

\section{INTRODUCTION}

Primary cardiac lymphoma (PCL) is defined as an extranodal lymphoma that involves only the heart and/or pericardium [1,2]. It is a rare cardiac tumor that is challenging to accurately diagnose. Although definitive diagnosis is possible by histopathologic examination (HPE), prior imaging assessment proves helpful. Transthoracic echocardiography (TTE) is usually the initial imaging technique. With advances in technology, cardiac magnetic resonance imaging (CMR) has allowed detailed assessment of tumor location, functional characteristics, and tissue characterization [3]. Computed tomography (CT) is often performed to evaluate other organs in patients with PCL. We report two cases of PCL presenting with typical and atypical features on CMR.

\section{CASE REPORT}

Our Institutional Review Board does not require ethical clearance for the publication of case reports. Informed consent has been obtained.

(c) This is an Open Access article distributed under the terms of the Creative Commons Attribution Non-Commercial License (http://creativecommons.org/licenses/bync/4.0) which permits unrestricted non-commercial use, distribution, and reproduction in any medium, provided the original work is properly cited.

\section{Case 1}

A 60-year-old lady presented with a 3-month history of dyspnea and constitutional symptoms. Electrocardiography (ECG) showed sinus rhythm. She was anemic with a hemoglobin level of $7.6 \mathrm{~g} / \mathrm{dL}$ (reference range 12-16 g/dL) and had elevated serum lactate dehydrogenase (LDH) of $409 \mathrm{U} / \mathrm{L}$ (reference range 140-280 U/L). TTE demonstrated a large right atrial tumor with pericardial effusion. Subsequent CMR revealed a solid right atrial tumor with a pericardial atrioventricular groove and right ventricular infiltration (Fig. 1A). Encasement of the right coronary artery (RCA) was evident (Fig. 1B). Heterogeneous enhancement was demonstrated following gadolinium administration. Pericardial effusion was also seen (Fig. 1C). Contrast CT scan of the thorax, abdomen, and pelvis confirmed no extra-cardiac involvement. An endomyocardial biopsy verified high grade B cell lymphoma. Chemotherapy was initiated. She received 1 cycle of rituximab, cyclophosphamide, doxorubicin, vincristine, and prednisone chemotherapy (R-CHOP); 4 cycles of rituximab, etoposide, prednisone, vincristine, cyclophosphamide, and doxorubicin (R-EPOCH) regime; and $2 \mathrm{cy}-$ cles of high dose methotrexate. Follow up CMR 6 months later showed significant reduction in tumor size (Fig. 1D). 


\section{Case 2}

A 44-year-old lady presented to the emergency department with a 3-day history of chest pain associated with heart failure symptoms. On examination, she was tachypneic and had bibasilar lung crepitation. Cardiac auscultation revealed a systolic murmur. Resting ECG demonstrated normal sinus rhythm. LDH
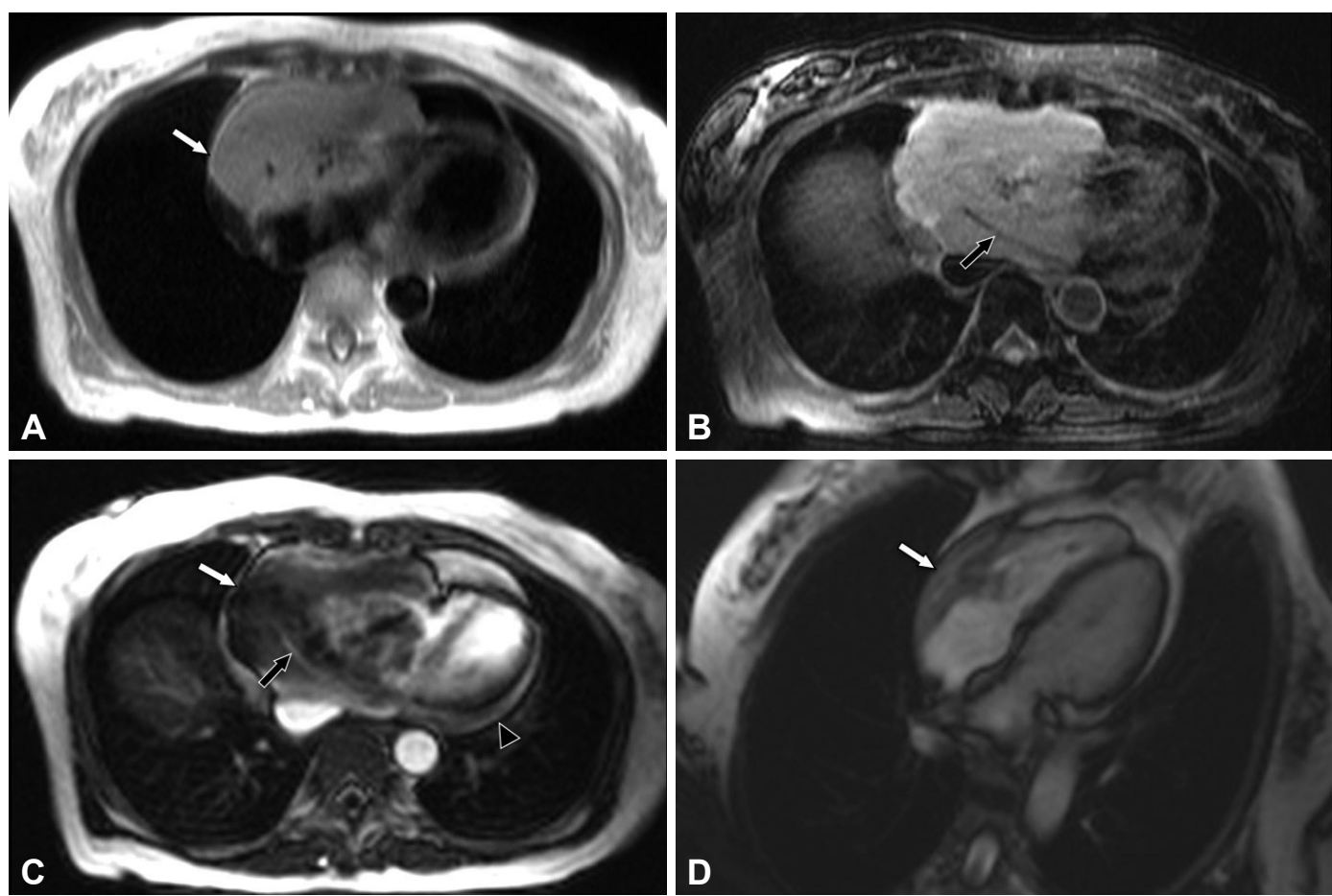

Fig. 1. Primary cardiac lymphoma in a 60-year-old lady who presented with dyspnea. CMR axial (A) half-Fourier acquisition single-shot turbo spin echo (B) T2-weighted fat suppression image (T2FS), and (C) early gadolinium phase-sensitive inversion recovery overview imaging were performed. An infiltrative mass involving the right atrium, right ventricle, atrioventricular groove, and pericardium (white arrows) appears heterogeneously hyperintense on T2FS and demonstrates heterogeneous enhancement in early gadolinium phase. Encasement of the right coronary artery (black arrows) and pericardial effusion (black arrow head) are also evident. (D) Six months later, follow up CMR (fourchamber view, steady state free precession) revealed significant reduction of tumor size (white arrow). CMR: cardiac resonance imaging.
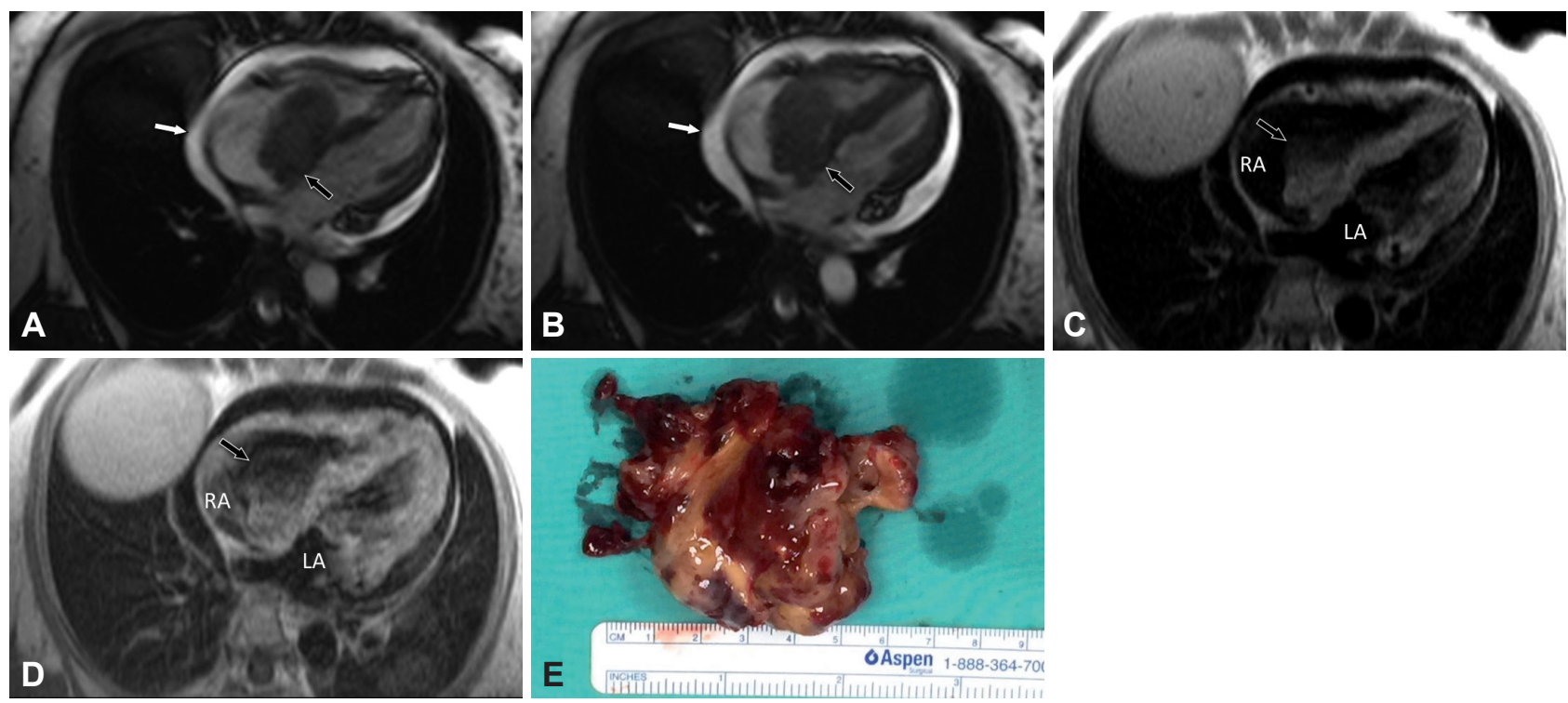

Fig. 2. A 44-year-old lady who presented with chest pain. Cardiac resonance imaging 4-chamber steady state free precession cine in diastole (A) and systole (B) showing a lobulated mobile mass arising from the inter-atrial septum (black arrows), protruding into the right ventricle during diastole. Pericardial effusion is seen (white arrows). Pre- (C) and post gadolinium (D) 4 chamber T1 weighted images were acquired, revealing a homogeneously isointense mass. Faint homogeneous enhancement similar to myocardial enhancement (black arrows) on post contrast T1 weighted image was observed. (E) Surgical pathology revealed primary cardiac lymphoma. 
was elevated (367 U/L). TTE showed a mobile right atrial mass, and CMR was arranged. Contrast CT scan of the thorax, abdomen, and pelvis confirmed no extra-cardiac involvement. On steady state free precession sequence, a large mobile mass arising from the right side of the inter-atrial septum was evident; which prolapsed into the right ventricle during diastole (Fig. 2A and B). Pericardial effusion was noted. The mass appears isointense to myocardium on pre-gadolinium $\mathrm{T} 1$ weighted imaging (T1WI) sequence (Fig. 2C) and demonstrates homogenous faint enhancement following contrast administration on T1WI (Fig. 2D). In view of the patient being hemodynamically unstable, an emergency open thoracotomy was embarked, revealing a pedunculated cardiac mass (Fig. 2E). HPE revealed diffuse large $\mathrm{B}$ cell Hodgkin's lymphoma. The peri-operative period was uneventful. The patient was referred to oncology and underwent $1 \mathrm{R}-\mathrm{CHOP}$ regime. Unfortunately, she died from sepsis secondary to pneumonia 6 months later.

\section{DISCUSSION}

PCL is rare, comprising a mere $1.3 \%$ of all primary cardiac tumors [1]. It usually affects the right heart chamber [2,4]. Dyspnea is the most common symptom, followed by chest pain and constitutional symptoms. PCL normally results in an arrhythmic pattern on ECG. Both of our patients presented with the above symptoms. Sinus rhythm was noted in both patients. $\mathrm{LDH}$ is increased in the majority of patients, as observed in our patients [2].

Due to its non-specific presentation, imaging plays a crucial role in diagnosis of PCL. TTE was and still is the primary imaging modality used to assess cardiac pathologies. However, the availability of CMR has allowed more detailed assessment of tumor location, functional characteristics, and tissue characterization [3]. This marked superiority of imaging information has made CMR a preferred and gold standard modality in further assessment of cardiac tumors.

The imaging features of PCL on CMR have been widely discussed in the literature. PCL most commonly presents as an invasive right heart chamber mass infiltrating the pericardium and is usually associated with pericardial effusion. Typically, it is isointense on T1 weighted, iso- to hyperintense on T2 weighted, and is not suppressed on fat suppression sequences. PCL demonstrates heterogeneous enhancement following gadolinium administration [3,4]. Encasement of the RCA, when present, favors PCL over angiosarcoma [5]. These features were noted in our first case.

Our second patient presented with atypical CMR features of PCL. She had a pedunculated mass attached to the right side of the inter-atrial septum. The mass was not invading nearby structures. It was noted to be mobile and prolapsed into the right ven- tricle during diastole, effectively obstructing the tricuspid valve. These features are classically seen in atrial myxoma $[3,6]$. However, atrial myxomas tend to be located on the left side and usually exhibit heterogeneous enhancement. Our patient, however, had a faintly homogenously enhancing lesion [6]. This feature initially led us astray in the diagnosis.

Both cases described above are good examples of overlapping features between certain cardiac tumors that contribute to a diagnostic dilemma when imaging with CMR. Both of our patients had a right-sided tumor, which is also a feature of angiosarcoma and lymphoma. However, angiosarcomas tend to affect a younger population, unlike our patients. Right ventricular involvement is a feature that supports the diagnosis of lymphoma rather than angiosarcoma. However, both of our patients had pericardial effusions at presentation, which is more commonly associated with angiosarcoma [5].

The mainstay of treatment for PCL is combined surgery and chemotherapy. Chin et al. [7] found that treatment with chemotherapy alone resulted in remission in almost $61 \%$ of patients, while surgery alone had no effect on outcome. Surgery buys time for chemotherapy to be of therapeutic value. Our first patient had significant reduction in tumor size following chemotherapy. The second patient underwent surgery prior to chemotherapy because she was hemodynamically compromised. Treatment should be tailored on a case-to-case basis.

In conclusion, due to its rarity, PCL is challenging to diagnose. CMR provides an avenue to accurately diagnose this condition prior to starting treatment. Knowledge of the typical and atypical features on CMR is of utmost importance to avoid potential pitfalls.

\section{Conflicts of Interest}

The authors declare that they have no conflict of interest.

\section{REFERENCES}

1. Johri A, Baetz T, Isotalo PA, Nolan RL, Sanfilippo AJ, Ropchan G. Primary cardiac diffuse large $B$ cell lymphoma presenting with superior vena cava syndrome. Can J Cardiol 2009;25:e210-e212.

2. Petrich A, Cho SI, Billett H. Primary cardiac lymphoma: an analysis of presentation, treatment, and outcome patterns. Cancer 2011;117:581-589.

3. O’Donnell DH, Abbara S, Chaithiraphan V, Yared K, Killeen RP, Cury RC, et al. Cardiac tumors: optimal cardiac MR sequences and spectrum of imaging appearances. Am J Roentgenol 2009;193:377-387.

4. Jeudy J, Kirsch J, Tavora F, Burke AP, Franks TJ, Mohammed TL, et al. From the radiologic pathology archives: cardiac lymphoma: radiologicpathologic correlation. Radiographics 2012;32:1369-1380.

5. Colin GC, Symons R, Dymarkowski S, Gerber B, Bogaert J. Value of CMR to differentiate cardiac angiosarcoma from cardiac lymphoma. JACC Cardiovasc Imaging 2015;8:744-746.

6. Grebenc ML, Rosado-de-Christenson ML, Green CE, Burke AP, Galvin JR. Cardiac myxoma: imaging features in 83 patients. Radiographics 2002; 22:673-689.

7. Chin JY, Chung MH, Kim JJ, Lee JH, Kim JH, Maeng IH, et al. Extensive primary cardiac lymphoma diagnosed by percutaneous endomyocardial biopsy. J Cardiovasc Ultrasound 2009;17:141-144. 